\title{
ON FINITELY EQUIVALENT CONTINUA
}

\author{
JANUSZ J. CHARATONIK
}

Received 7 January 2002

\begin{abstract}
For positive integers $m$ and $n$, relations between (hereditary) $m$ - and $n$-equivalence are studied, mostly for arc-like continua. Several structural and mapping problems concerning (hereditarily) finitely equivalent continua are formulated.
\end{abstract}

2000 Mathematics Subject Classification: 54F15.

A continuum means a compact connected metric space. For a positive integer $n$, a continuum $X$ is said to be $n$-equivalent provided that $X$ contains exactly $n$ topologically distinct subcontinua. A continuum $X$ is said to be hereditarily $n$-equivalent provided that each nondegenerate subcontinuum of $X$ is $n$-equivalent. If there exists a positive integer $n$ such that $X$ is $n$-equivalent, then $X$ is said to be finitely equivalent. Thus, for $n=1$, the concepts of "1equivalent" and "hereditarily 1-equivalent" coincide, and they mean the same as "hereditarily equivalent" in the sense considered, for example, by Cook in [2].

Observe the following statement.

STATEMENT 1. Each subcontinuum of an $n$-equivalent continuum is $m$ equivalent for some $m \leq n$. Thus, each finitely equivalent continuum is hereditarily finitely equivalent.

Some structural results concerning finitely equivalent continua are obtained by Nadler Jr. and Pierce in [9]. They have shown that if a continuum $X$ is (a) semi-locally connected at each of its noncut points, then it is finitely equivalent if and only if it is a graph; (b) aposyndetic at each of its noncut points and finitely equivalent, then it is a graph. Furthermore, in both cases (a) and (b), if $X$ is $n$-equivalent, then each subcontinuum of $X$ is a $\theta_{n+1}$-continuum. Recall that Nadler Jr. and Pierce in [9, page 209] posed the following problem.

Problem 2. Determine which graphs, or at least how many, are $n$-equivalent for each $n$.

The arc and the pseudo-arc are the only known 1-equivalent continua. In [10] Whyburn has shown that each planar 1-equivalent continuum is tree-like, and planarity assumption has been deleted after 40 years by Cook [2] who proved tree-likeness of any 1-equivalent continuum. But it is still not known whether or not the arc and the pseudo-arc are the only ones among 1-equivalent continua.

In contrast to 1-equivalent case, 2-equivalent continua need not be hereditarily 2-equivalent, a simple closed curve is 2-equivalent while not hereditarily 
2-equivalent. The 2-equivalent continua were studied by Mahavier in [5] who proved that if a 2-equivalent continuum contains an arc, then it is a simple triod, a simple closed curve or irreducible, and that the only locally connected 2-equivalent continua are a simple triod and a simple closed curve. It is also shown that if $X$ is a decomposable, not locally connected, 2-equivalent continuum containing an arc, then $X$ is arc-like and it is the closure of a topological ray $R$ such that the remainder $\operatorname{cl}(R) \backslash R$ is an end continuum of $X$. Furthermore, two examples of 2-equivalent continua are presented in [5]: the first, [5, Example 1, page 246], is a decomposable continuum $X$ which is the closure of a ray $R$ such that the remainder $\operatorname{cl}(R) \backslash R$ is homeomorphic to $X$; the second, [5, Example 2, page 247], is an arc-like hereditarily decomposable continuum containing no arc.

Looking for an example of a hereditarily 2-equivalent continuum note that the former example surely is not hereditarily 2-equivalent because it contains an arc. We analyze the latter one.

The continuum $M$ constructed in [5, Example 2, page 247] does not contain any arc, and it contains a continuum $N$ such that each subcontinuum of $M$ is homeomorphic to $M$ or to $N$, see [5, the paragraph following Lemma 3, page 249]. Further, by its construction, $N$ does contain continua homeomorphic to $M$ (see [5, the final part of the proof, page 251]). Therefore, the following statement is established.

THEOREM 3. The continuum $M$ constructed in [5, Example 2, page 247] has the following properties:

(a) $M$ is an arc-like;

(b) $M$ is hereditarily decomposable;

(c) $M$ does not contain any arc;

(d) $M$ is hereditarily 2-equivalent.

In connection with the above theorem, the following problem can be posed.

Problem 4. Determine for what integers $n \geq 3$, there exists a continuum $M$ satisfying conditions (a), (b), and (c) of Theorem 3 and being hereditarily $n$-equivalent.

The following results are consequences of [1, Theorem, page 35].

THEOREM 5. For each hereditarily n-equivalent continuum $X$, that does not contain any arc, there exists an $(n+2)$-equivalent continuum $Y$ such that each of its subcontinua is homomorphic either to a subcontinuum of $X$ or to $Y$, or to an arc.

Proof. Indeed, a compactification $Y$ of a ray $R$ having the continuum $X$ as the remainder, that is, such that $X=\operatorname{cl}(R) \backslash R$ is such a continuum.

Since if $M$ is arc-like and hereditarily decomposable, then so is any of compactifications $Y$ of a ray having the continuum $X$ as the remainder, we get the next result as a consequence of Theorem 5 . 
COROLlary 6. If a continuum $M$ satisfies conditions (a), (b), and (c) of Theorem 3 and is hereditarily n-equivalent, then any of compactifications of a ray having the continuum $M$ as the remainder satisfies conditions ( $a$ ) and (b) of Theorem 3 and is $(n+2)$-equivalent.

In [7], an uncountable family $\mathscr{F}$ is constructed of compactifications of the ray with the remainder being the pseudo-arc.

STATEMENT 7. Each member $X$ of the (uncountable) family $\mathscr{F}$ constructed in [7] is an arc-like 3-equivalent continuum. Any subcontinuum of $X$ is homeomorphic to an arc, to a pseudo-arc, or to the whole $X$.

A continuum $X$ has the $R N T$-property (retractable onto near trees) provided that for each $\varepsilon>0$, there exists a $\delta>0$ such that if a tree $T$ is $\delta$-near to $X$ with respect to the Hausdorff distance, then there is an $\varepsilon$-retraction of $X$ onto $T$, see [6, Definition 0]. It is shown in [6, Theorem 5] that if a continuum $X$ is a compactification of the ray $R$ and $X$ has the RNT-property, then the remainder $\operatorname{cl}(R) \backslash R \subset X=\operatorname{cl}(R)$ is the pseudo-arc. Therefore, Theorem 5 implies the following proposition.

Proposition 8. Each compactification $X$ of the ray having the RNT-property is a 3-equivalent continuum. Each subcontinuum of $X$ is homeomorphic to an arc, a pseudo-arc, or to the whole $X$.

Observe that $M$ of Theorem 3 being an arc-like is hereditarily unicoherent, and being hereditarily decomposable, it is a $\lambda$-dendroid (containing no arc). Another (perhaps the first) example of a $\lambda$-dendroid, in fact, an arc-like, containing no arc, has been constructed by Janiszewski in 1912, [3] but his description was rather intuitive than precise. It would be interesting to investigate if that old example of Janiszewski is or is not $n$-equivalent (hereditarily $n$-equivalent) for some $n$.

The following problems can be considered as a program of a study in the area rather than particular questions.

Problems 9. For each positive integer $n$, characterize continua which are (a) $n$-equivalent; (b) hereditarily $n$-equivalent.

Problem 10. Characterize continua which are finitely equivalent.

Sometimes a characterization of a class of spaces (or of spaces having a certain property) can be expressed in terms of containing some particular spaces. A classical illustration of this is a well-known characterization of nonplanar graphs by containing the two Kuratowski's graphs: $K_{5}$ and $K_{3,3}$, see, for example, [8, Theorem 9.36, page 159]. To be more precise, recall the following concept. Let $\mathscr{A}$ be a class of spaces and let $\mathscr{P}$ be a property. Then $\mathscr{P}$ is said to be finite (or countable) in the class $\mathscr{A}$ provided that there is a finite (or countable, 
respectively) set $\mathscr{S}$ of members of $\mathscr{A}$ such that a member $X$ has the property $\mathscr{P}$ if and only if $X$ contains a homeomorphic copy of some member of $\mathscr{Y}$. The result of [7] mentioned above in Statement 7 shows that this is not the way of characterizing 3-equivalent continua. Namely, the existence of the family $\mathscr{F}$ shows the following theorem.

THEOREM 11. The property of being 3-equivalent is neither finite nor countable in the class of (a) all continua; (b) arc-like continua.

A mapping $f: X \rightarrow Y$ between continua $X$ and $Y$ is said to be

(i) atomic provided that for each subcontinuum $K$ of $X$, either $f(K)$ is degenerate or $f^{-1}(f(K))=K$;

(ii) monotone provided that the inverse image of each subcontinuum of $Y$ is connected;

(iii) hereditarily monotone provided that for each subcontinuum $K$ of $X$, the partial mapping $f \mid K: K \rightarrow f(K)$ is monotone.

It is known that each atomic mapping is hereditarily monotone, see, for example, [4, (4.14), page 17]. Since each arcwise connected 2-equivalent continuum is either a simple closed curve or a simple triod, see [5, Theorem 2, page 244], each semilocally connected 3-equivalent continuum is either a simple 4-od [8, Definition 9.8, page 143] (i.e., a letter X) or a letter $\mathrm{H}$, see [9, page 209]. And since these continua are preserved under atomic mappings (as it is easy to see), we conclude that atomic mappings preserve the property of being 2-equivalent and being 3-equivalent for locally connected continua. However, this is not an interesting result, because each atomic mapping of an arcwise connected continuum onto a nondegenerate continuum is a homeomorphism, see [4, (6.3), page 51]. But the result cannot be extended to hereditarily monotone mappings, because a mapping that shrinks one arm of a simple triod to a point is hereditarily monotone and not atomic, and it maps a 2-equivalent continuum onto an arc that is 1-equivalent. On the other hand, if $X$ is the 2equivalent continuum which is the closure of a ray $R$ as described in [5, Example 1, page 246], then the mapping $f: X \rightarrow[0,1]$, that shrinks the remainder $\operatorname{cl}(R) \backslash R$ to a point (and is a homeomorphism on $R$ ), is atomic and it maps 2-equivalent continuum $X$ onto the 1-equivalent continuum $[0,1]$. Therefore, atomic mappings do not preserve the property of being a 2-equivalent continuum. In connection with these examples, the following question can be asked.

QUESTION 12. Let a continuum $X$ be $n$-equivalent and let a mapping $f$ : $X \rightarrow Y$ be an atomic surjection. Must then $Y$ be $m$-equivalent for some $m \leq n$ ?

In general, we can pose the following problems.

Problems 13. What kinds of mappings between continua preserve the property of being: (a) $n$-equivalent? (b) hereditarily $n$-equivalent? (c) finitely equivalent? 


\section{REFERENCES}

[1] J. M. Aarts and P. van Emde Boas, Continua as remainders in compact extensions, Nieuw Arch. Wisk. (3) 15 (1967), 34-37.

[2] H. Cook, Tree-likeness of hereditarily equivalent continua, Fund. Math. 68 (1970), 203-205.

[3] Z. Janiszewski, Über die Begriffe "Linie" und "Flache", Proceedings of the Fifth International Congress of Mathematicians, Cambridge, vol. 2, Cambridge University Press, 1912, pp. 126-128, reprinted in Oeuvres Choisies, Państwowe Wydawnictwo Naukowe, Warsaw, 1962, pp. 127-129 (German).

[4] T. Maćkowiak, Continuous mappings on continua, Dissertationes Math. (Rozprawy Mat.) 158 (1979), 1-91.

[5] W. S. Mahavier, Continua with only two topologically different subcontinua, Topology Appl. 94 (1999), no. 1-3, 243-252.

[6] V. Martínez-de-la-Vega, The RNT property on compactifications of the ray, Continuum Theory: (A. Illanes, S. Macías and W. Lewis, eds.), Lecture Notes in Pure and Applied Mathematics, vol. 230, Marcel Dekker, New York, pp. 211-227, 2002.

[7]___ An uncountable family of metric compactifications of the ray with remainder pseudo-arc, preprint, 2002.

[8] S. B. Nadler Jr., Continuum Theory. An Introduction, Monographs and Textbooks in Pure and Applied Mathematics, vol. 158, Marcel Dekker, New York, 1992.

[9] S. B. Nadler Jr. and B. Pierce, Finitely equivalent continua semi-locally-connected at non-cut points, Topology Proc. 19 (1994), 199-213.

[10] G. T. Whyburn, A continuum every subcontinuum of which separates the plane, Amer. J. Math. 52 (1930), 319-330.

Janusz J. Charatonik: Instituto de Matemáticas, UNAM, Circuito Exterior, Ciudad Universitaria, 04510 México DF, Mexico

E-mail address: jjc@math. unam.mx 


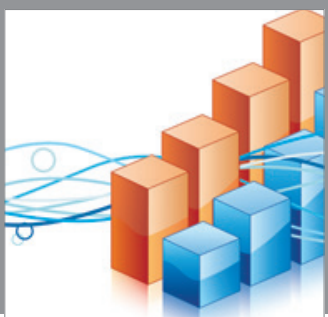

Advances in

Operations Research

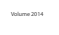

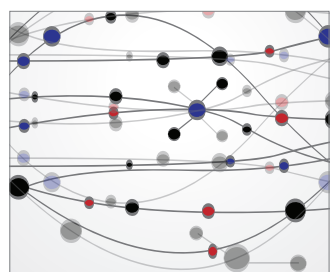

\section{The Scientific} World Journal
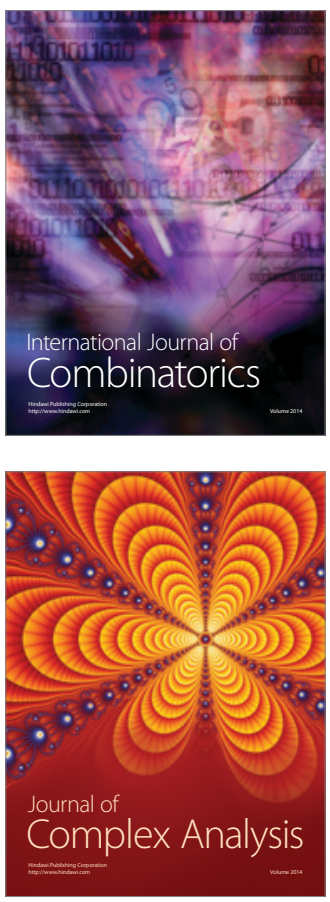

International Journal of

Mathematics and

Mathematical

Sciences
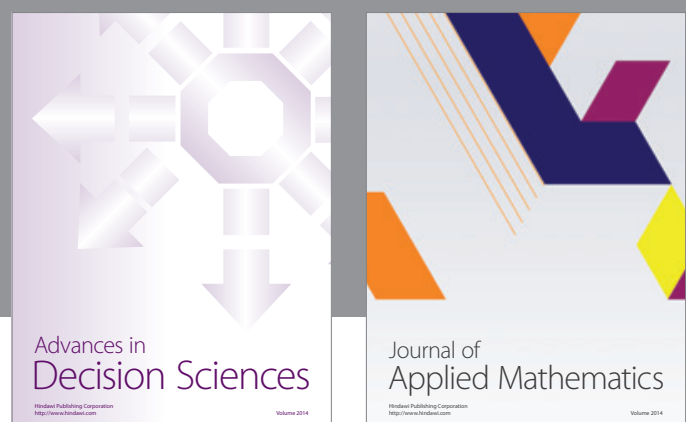

Journal of

Applied Mathematics
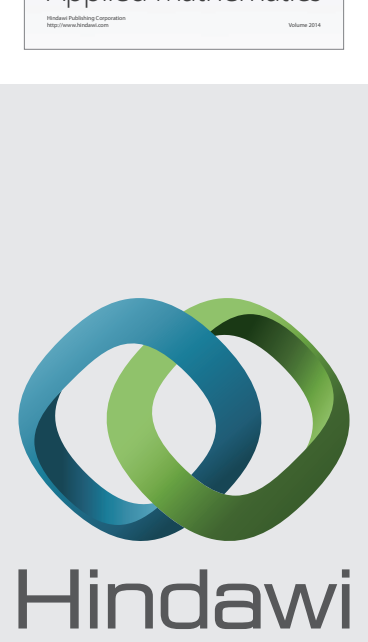

Submit your manuscripts at http://www.hindawi.com
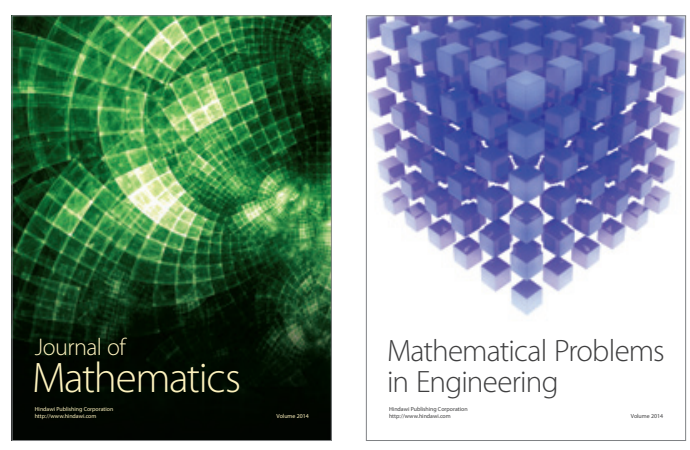

Mathematical Problems in Engineering
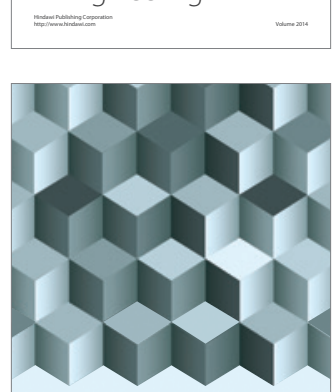

Journal of

Function Spaces
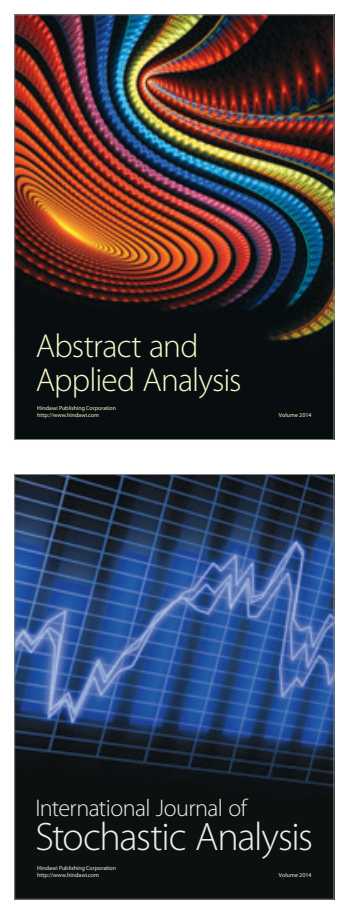

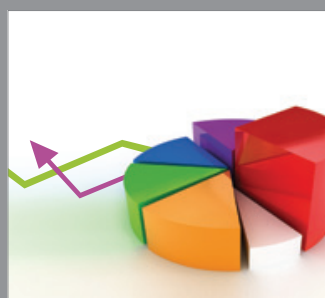

ournal of

Probability and Statistics

Promensencen
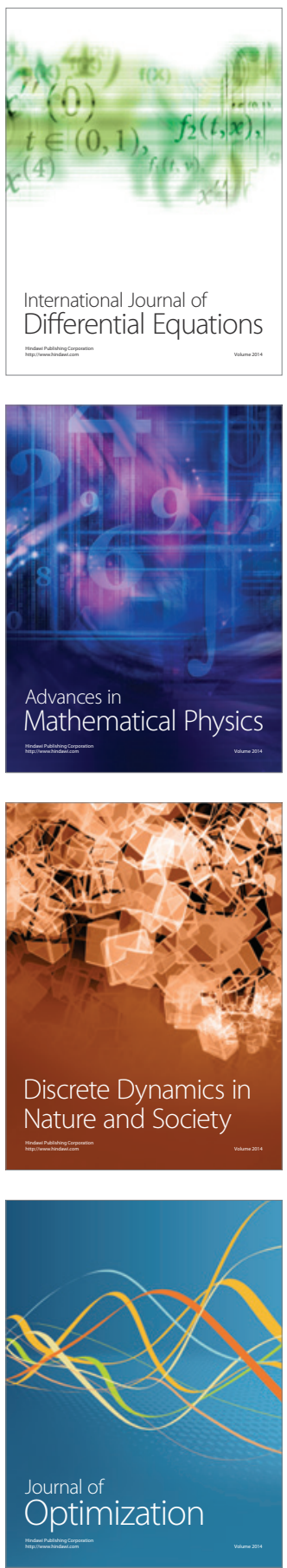